\title{
VALOR PRONÓSTICO EN LA PROSTATECTOMÍA RADICAL DEL NÚMERO DE CILINDROS POSITIVOS Y PORCENTAJE DE CÁNCER POR CILINDRO DE LA BIOPSIA PROSTÁTICA
}

\author{
F. OSORIO PAZO, L. MOURO GIUDICE, J. JUBIN MARTÍNEZ
}

Cátedra de Urología. Hospital de clinicas "Dr. Manuel Quintela". Montevideo. Uruguay.

Actas Urol Esp. 27 (7): 538-542, 2003

\section{RESUMEN}

VALOR PRONÓSTICO EN LA PROSTATECTOMÍA RADICAL DEL NÚMERO DE CILINDROS POSITIVOS Y PORCENTAJE DE CÁNCER POR CILINDRO DE LA BIOPSIA PROSTÁTICA

OBJETIVO: El Score de Gleason de la biopsia, la dosificación del antígeno prostático específico y el estadio clínico son factores pronósticos independientes bien conocidos. Desde la popularización del uso del antígeno prostático específico, se ha visto una migración de los estadios clínicos de los pacientes con cáncer de próstata, siendo la realidad actual pacientes con estadios precoces, antígeno bajo y tumores bien diferenciados. Esto ha impulsado la búsqueda de otros factores pronósticos para estratificar el riesgo de los mismos. Nosotros analizaremos el valor pronóstico de la cuantificación histológica en los pacientes sometidos a prostatectomía radical.

MATERIAL Y MÉTODOS: De una serie de 112 prostatectomías radicales realizadas por el mismo urólogo, se seleccionan 81 en las cuales se contó con la cuantificación tumoral en el informe patológico.

RESULTADOS: Al comparar los grupos de hasta 2 cilindros positivos con el de más de 4, se multiplicó por, 2,4 el riesgo de margen positivo (15,4\% vs. $37,5 \%$ ) y duplicó el riesgo de recaída bioquímica (34\% vs. $60 \%$ ). Del mismo modo, al analizar el valor pronóstico del porcentaje de cáncer por cilindro, se vio que al comparar la presencia de menos de $20 \%$ a más de $40 \%$ de cáncer por cilindro, multiplicó por 5 el riesgo de margen positivo $(8,7 \%$ vs. $44,1 \%)$ y triplicó el riesgo de recaída bioquímica ( $15 \%$ vs. $48 \%$ ).

CONCLUSIONES: Tanto la cantidad de cilindros positivos, el porcentaje de cilindros positivos del total de la biopsia y el porcentaje de cáncer de cada cilindro demostraron ser factores pronósticos predictivos significativos que deberían ser analizados en todos los pacientes que son sometidos a una biopsia prostática, para de este modo poder estratificar mejor el riesgo de estos pacientes y así ayudar a planificar una más adecuada estrategia de tratamiento.

PALABRAS CLAVE: Cáncer de próstata. Factores pronósticos. Prostatectomía radical retropúbica.

\section{ABSTRACT}

PROGNOSTIC VALUE IN RADICAL PROSTATECTOMY OF POSITIVE CILINDERS NUMBER AND CANCER PERCENTAGE PER CILINDER IN PROSTATE BIOPSY

INTRODUCTION: Clinical stage, PSA value and Gleason score in biopsy are well known independent prognostic factors in prostate cancer. Since the widespread use of PSA, we assist to a changing trend in clinical presentation of patients with prostate cancer toward low stage, low PSA and well-differentiated tumors. This has motivated the search of new prognostic factors to predict those patients at risk of poor outcome. We analyze the histological quantification of tumor in biopsy as a prognostic factor in patients that underwent radical retropubic prostatectomy (RRP).

MATERIALS AND METHODS: Of a series of 112 RRP performed by the same surgeon, we selected 81 in which tumor quantification at biopsy was assessed.

RESULTS: Comparing the group with up to 2 positive cores against the group with 4 or more, the risk of positive surgical margins in RRP multiplies by 2.4 (15.4\% vs. 37.5\%), and the risk of biochemical progression almost duplicates (34\% vs. 60\%). When percentage of tumor per core is analyzed, the presence of $40 \%$ or more per core compared to less than $20 \%$, multiplies the risk of positive margins by 5 ( $44.1 \%$ vs. $8.7 \%$ ), and the risk of biochemical relapse by 3 ( $48 \%$ vs. $15 \%)$.

CONCLUSIONS: The total number of positive cores, the percentage of the total of positive cores and the amount of cancer in each core, all demonstrated to be significant predictive prognostic factors that should be analyzed in every patient undergoing prostate biopsy, to predict the risk of poor outcome and therefore help planning the most adequate treatment strategy.

KEY WORDS: Prostate cancer. Prognostic factors. Radical retropubic prostatectomy. 
$\mathrm{L}^{\mathrm{a}}$ a prostatectomía radical aumenta claramente su eficacia cuando la enfermedad se encuentra en un estadio órgano o espécimen confinado ${ }^{1-4}, \mathrm{y}$ de este modo, la capacidad de predecir adecuadamente el estadio patológico final a partir de factores preoperatorios, es de gran importancia en la selección de aquellos pacientes que obtendrán un mayor beneficio de la cirugía ${ }^{5}$. El Score de Gleason de la biopsia, la dosificación del antígeno prostático específico (APE) y el estadio clínico son factores pronósticos independientes bien conocidos y de indiscutible valor al encarar el tratamiento de un paciente portador de cáncer de próstata ${ }^{6-10}$.

En los últimos años se ha observado una migración de los estadios clínicos de los pacientes con cáncer de próstata, siendo la realidad actual pacientes con estadios precoces, antígeno bajo y tumores bien diferenciados, seguramente vinculado al uso cada vez más extendido del antígeno prostático específico ${ }^{11,12}$. Pero a pesar de esta migración hacia una enfermedad clínicamente organoconfinada, a una proporción importante de pacientes (40-60\%) se les detecta enfermedad extracapsular luego de la prostatectomía radical y entre un 30 a $40 \%$ de los pacientes tendrán una recurrencia de la enfermedad dentro de los 10 años luego de la cirugía ${ }^{13}$. Estos resultados han impulsado la búsqueda de otros factores pronósticos para poder estratificar mejor a este grupo de pacientes con enfermedad clínicamente organoconfinada.

La biopsia prostática transrectal por sextante guiada por ecografía es una estrategia de más de diez años de aplicación, pero que ha sido desplazada por protocolos con un número extendido de tomas y que prioriza la importancia.

\section{RESULTADOS}

De los 81 pacientes de la serie, el número de cilindros con cáncer fue de 1 a 11 (promedio: 2,8), el porcentaje de cilindros con cáncer del total fue de la topografía de las mismas ${ }^{14,15}$. Asi mismo, el cúmulo de información que se obtiene del análisis anatomopatológico de dichas biopsias, es mucho mayor que en sus comienzos, no sólo en cuanto al diagnóstico del cáncer de próstata, sino que también identificando pacientes de riesgo en los cuales estaría indicada la re-biopsia y en aquellos con diagnóstico confirmados obtener de la misma fac- tores pronósticos de utilidad para ayudar a definir un tratamiento adecuado ${ }^{16-19}$. es así que nosotros analizaremos el valor pronóstico de la cuantificación histológica de la biopsia prostática para pacientes con cáncer clínicamente organoconfinado, a los cuales se les sometió a prostatectomía radical.

\section{MATERIALES Y MÉTODOS}

Se trata del análisis retrospectivo de una serie de 112 pacientes a los cuales se les realizó, por el mismo urólogo, una prostatectomía radical por cáncer de próstata, de los cuales se seleccionaron 81, siendo el criterio de exclusión la falta de la cuantificación histológica de cáncer en el informe anatomopatológico de la biopsia. La edad media de la muestra fue de 64,3 años (49 a 73), el APE medio de 7,30 ng/ml (3,2 a 26,5), y los estadios clínicos preoperatorios fueron: T1c: $64,2 \%$, T2a: $34,57 \%$ y $\mathrm{T} 2 \mathrm{~b}: 1,23 \%$. El número promedio de cilindros por biopsia fue de 6,15 (3 a 12).

Se analizó el valor en la incidencia de márgenes positivos y la recaída bioquímica precoz (dentro de los 6 primeros meses), del número de cilindros positivos para cáncer, el porcentaje de cilindros positivos y el porcentaje de cáncer por cilindro de la biopsia prostática. Se definió un aumento del APE mayor a $0,1 \mathrm{ng} / \mathrm{ml}$ como recaída bioquímica, de 8 a 100\%, y el porcentaje de cáncer por cilindro de 5 a 95\%. Globalmente, hubo una incidencia de $75,9 \%$ de márgenes negativos, ninguno de los pacientes presentó ganglios positivos en la linfadenectomía, y permanecieron libres de recaída bioquímica hasta los 6 meses el 82,1\%. En la Tabla I se resumen los resultados patológicos globales.

$\mathrm{Al}$ analizar el riesgo de márgenes positivos, se observaron los siguientes resultados: con 1 a 2,3 a 4 y más de 4 cilindros con cáncer, la incidencia fue de 15,38\%, 31,25\% y 37,5\%, respectivamente.

Del mismo modo, se vio un aumento de la incidencia al aumentar el porcentaje de cáncer por cilindro de hasta $20 \%$, entre 20 y $40 \%$ y más de $40 \%(8,7 \%, 20,83 \%$ y $44,12 \%$ de margen positivo). El porcentaje de cilindros positivos también mostró un valor predictivo de significación, con 0\% de márgenes positivos cuando menos del 20\% de los cilindros estaban comprometidos, frente a $38 \%$ cuando más del $60 \%$ eran positivos. 
TABLA I

\begin{tabular}{|c|c|}
\hline Margen & \\
\hline 0 margen positivo & $75,89 \%$ \\
\hline 1 margen positivo & $15,18 \%$ \\
\hline 2 margen positivo & $7,14 \%$ \\
\hline 3 margen positivo & $1,79 \%$ \\
\hline Estadio patológico & \\
\hline pT2a & $23,46 \%$ \\
\hline pT2b & $46,91 \%$ \\
\hline pT3a & $14,81 \%$ \\
\hline pT3b & $14,81 \%$ \\
\hline pNO & $100 \%$ \\
\hline APE $\varangle \mathbf{0} \mathbf{~ n g / ~} \mathbf{~ m} *$ & $82,1 \%$ \\
\hline
\end{tabular}

*Hasta 6 meses post-operatorio.

El riesgo de recaída bioquímica precoz (hasta los 6 meses), también se modificó claramente al comparar los resultados con los elementos pronósticos analizados. La misma pasó de 34\% cuando habían hasta 2 cilindros comprometidos a $60 \%$ con más de 4 . Así mismo, la incidencia de recaída aumentó del 15\% al 48\% cuando se comparan aquellos pacientes con hasta $20 \%$ de cáncer por cilindro con aquellos con más del $40 \%$. En cuanto al porcentaje de cilindros comprometidos del total de la biopsia, ésta no mostró una incidencia significativa, al aumentar de $25 \%$ a $33 \%$ el riesgo de recaída con hasta $20 \%$ de los cilindros positivos frente a más del $60 \%$ de los mismos comprometidos (Tabla II) (Fig. 1).

\section{DISCUSIÓN}

En los últimos años se ha observado una migración de los estadios clínicos de los pacientes con cáncer de próstata, siendo la realidad actual pacientes con estadios precoces, antígeno bajo y tumores bien diferenciados ${ }^{11,12}$, y por lo tanto, candidatos a tratamientos pretendidamente curativos. Esto impulsó la búsqueda de nuevos factores pronósticos para mejor estratificar el riesgo en este grupo de pacientes, y así poder predecir más adecuadamente el riesgo de recidiva luego del tratamiento elegido. Dentro de este grupo de nuevos factores pronósticos, se encuentran los que surgen del análisis anatomopatológico de la biopsia
TABLA II

\begin{tabular}{|l|c|c|}
\hline № de cilindros + & Margen + & $\begin{array}{c}\text { Recaída } \\
\text { bioquímica }\end{array}$ \\
\hline 1 a 2 & $15,38 \%$ & $34,04 \%$ \\
\hline 3 a 4 & $31,25 \%$ & $28,21 \%$ \\
\hline Más de 4 & $37,50 \%$ & $60,00 \%$ \\
\hline \% de cáncer por cilindro \\
\hline Hasta $20 \%$ & $8,70 \%$ & $15,00 \%$ \\
\hline Entre 20 y $40 \%$ & $20,83 \%$ & $28,57 \%$ \\
\hline Más de $40 \%$ & $44,12 \%$ & $48,15 \%$ \\
\hline$\%$ positivas & $0 \%$ & $25 \%$ \\
\hline Hasta $20 \%$ & $21 \%$ & $29 \%$ \\
\hline 20 a $40 \%$ & $43 \%$ & $30 \%$ \\
\hline 40 a $60 \%$ & $38 \%$ & $33 \%$ \\
\hline Más de $60 \%$ & & \\
\hline
\end{tabular}

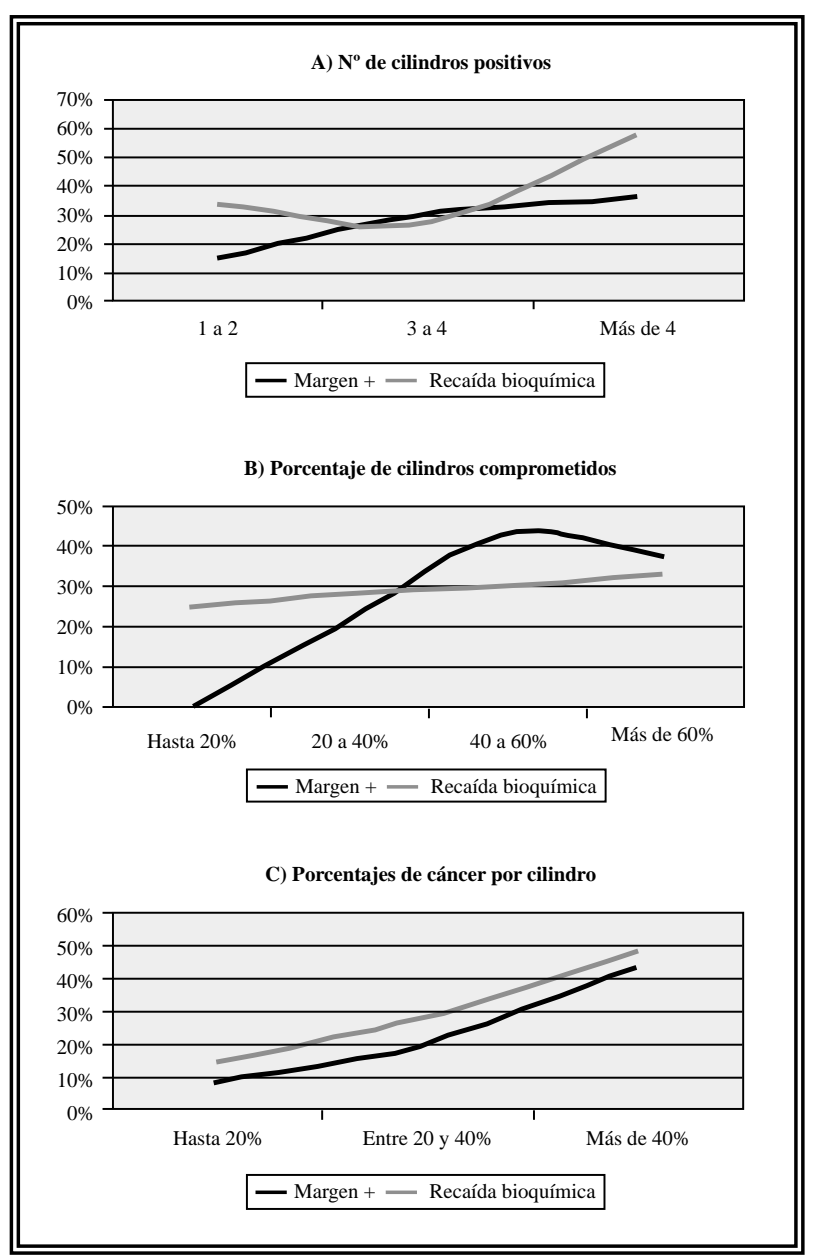

FIGURA 1 
prostática $^{20-24}$. Es un tema controversial si estos factores pronósticos secundarios que surgen de la cuantificación histológica de la biopsia prostática, tienen un valor predictivo independiente en cuanto a hallazgos patológicos adversos o falla bioquímica.

Boccon-Gibod L. y cols. ${ }^{25}$ y Aronson W.J. y cols. ${ }^{23}$, publican 2 series retrospectivas en las cuales muestran al porcentaje de cáncer por cilindro como el factor predictivo de mayor significación en cuanto a márgenes positivos y falla bioquímica incluso más aún que el Gleason o el APE preoperatorio.

En nuestra serie, si bien no comparamos los resultados con los factores pronósticos clásicos, nos permite sacar algunas conclusiones de utilidad. Encontramos un fuerte valor predictivo en el porcentaje de cáncer por cilindro, viendo que se quintuplicó el riesgo de márgenes positivos cuando ese porcentaje pasó de menos de $20 \%$ a más de $40 \%$, así como se triplicó el riesgo de recaída bioquímica precoz al comparar ese mismo rango. También, tanto el número de cilindros comprometidos como el porcentaje de cilindros del total de la biopsia, mostraron ser factores pronósticos importantes, destacándose en este sentido un $60 \%$ de recaída bioquímica y casi un $40 \%$ de margen positivo cuando se encontraba cáncer en más de 4 cilindros si se lo compara con $34 \%$ de recaída bioquímica y $15 \%$ de margen positivo cuando el número de cilindros era menor o igual a 2 .

\section{CONCLUSIONES}

Tanto la cantidad de cilindros positivos, el porcentaje de cilindros positivos del total de la biopsia y el porcentaje de cáncer de cada cilindro demostraron ser factores pronósticos predictivos significativos que deberían ser analizados en todos los pacientes que son sometidos a una biopsia prostática, para de este modo poder estratificar mejor el riesgo de estos pacientes y así ayudar a planificar una más adecuada estrategia de tratamiento.

\section{REFERENCIAS}

1. BLUTE ML, NATIV O, ZINCKE $\mathrm{H}$ et al.: Pattern of failure alter radical retropubic prostatectomy for clinically and pathologically localized adenocarcinoma of the prostate: influence of tumor deoxyribonucleic acid ploidy. J Urol 1989; 142: 1262.
2. PAULSON DF.: Impact of radical prostatectomy in the management of clinically localized disease. $J$ Urol 1994; 2 (152): 1826.

3. STEIN A, deKERNION JB, SMITH RB et al.: Prostate specific antigen levels after radical prostatectomy in patients with organ confined and locally extensive prostate cancer. $J$ Urol 1992; 2 (147): 942.

4. EPSTEIN JI, PIZOV G, WALSH PC.: Correlation of pathologic findings with progression following radical retropubic prostatectomy. Cancer 1993; 71: 3582.

5. EPSTEIN JI, WALSH PC, CARMICHAEL M et al.: Pathologic and clinical findings to predict tumor extent of nonpalpable (stage T1c) prostate cancer. JAMA 1994; 271: 368.

6. PARTIN AW, YOO J, CARTER HB et al.: The use of prostate specific antigen, clinical state and Gleason score to predict pathological stage in men with localized prostate cancer. J Urol 1993; 150: 110.

7. DUGAN JA, BOSTWICK DG, MYERS RP et al.: The definition and preoperative prediction of clinically insignificant prostate cancer. JAMA 1996; 275: 288.

8. PARTIN AW, KATTAN MW, SUBONG EN et al.: Combination of prostate-specific antigen, clinical stage, and Gleason score to predict pathological stage of localized prostate cancer. A multiinstitutional update. JAMA 1997; 277: 1445.

9. CATALONA WJ, SMITH DS, RATLIFF TL et al.: Detection of organ-confined prostate cancer is increased through prostatespecific antigen-based screening. JAMA 1993; 270: 948.

10. D'AMICO AV, WHITTINGTON R, MALKOWICZ SB, WEINSTEIN M, TOMASZEWSKI JE, SCHULTZ D, RICHIE JP et al.: Predicting prostate specific antigen outcome preoperatively in the prostate specific antigen era. J Urol 2001; 166: 2185-2188.

11. STEPHENSON RA.: Population-based prostate cancer trends in the PSA-era: data from the Surveillance, Epidemiology, and End Results (SEER). Program Monogr Urol 1998; 19: 1.

12. SUN L, GANCARCZYK K, PAQUETTE E et al.: Introduction to department of defense center for prostate disease research multicenter national prostate cancer database, and analysis of changes in the PSA-era. Urol Oncol 2001; 6: 203.

13. MOUL JW, CARROLL PR et al.: Predicting risk of prostate specific antigen recurrence after radical prostatectomy with the center for prostate disease research and cancer of the prostate strategic urologic research endeavor databases. J Urol 2001; 166: 1322.

14. BAUER JJ, ZENG J, ZHANG W et al.: Lateral biopsies added to the traditional sextant prostate biopsy pattern increases the detection rate of prostate cancer. Prostate Cancer Prostatic Dis 2000; 3: 43.

15. EPSTEIN JI et al.: Does increased needle biopsy sampling of the prostate detect a higher number of potentially insignificant tumors?. J Urol 2001; 166: 2181-2184.

16. WEINSTEIN MH, EPSTEIN JI.: Significance of highgrade prostatic intraepithelial neoplasia on needle biopsy. Hum Pathol 1993; 24: 624. 
17. CHEVILLE JC, REZNICEK MK, BOSTWICK DG.: The focus of "atypical glands suspicious for malignancy" in prostate needle biopsy specimens: incidence, histologic features, and clinical follow-up of cases diagnosed in a community practice. Am $J$ Clin Pathol 1997; 108: 633.

18. RENSHAW AA, SANTIS WF, RICHIE JP.: Clinicopathological characteristics of prostatic adenocarcinoma in men with atypical prostate needle biopsies. J Urol 1998; 159: 2018.

19. EPSTEIN JI, POTTER S.: The pathological interpretation and significance of prostate needle biopsy findings: implications and current controversies. $J$ Urol 2001; 166: 402-410.

20. D'AMICO AV, WHITTINGTON R, MALKOWICZ SB et al.: Clinical utility of the percentage of positive prostate biopsies in defining biochemical outcome after radical prostatectomy for patients with clinically localized prostate cancer. J Clin Oncol 2000; 18: 1164 .

21. PARTIN AW et al.: Prediction of prostate carcinoma stage by quantitative biopsy pathology. Cancer 2001; 91: 2322.
22. MOUL JW.: Prostate biopsy quantitative histology as a staging and prognostic factor. J Urol 2002; 167: 526-527.

23. ARONSON WJ et al.: Percent prostate needle biopsy tissue with cancer is more predictive of biochemical failure or adverse pathology after radical prostatectomy than prostate specific antigen or Gleason score. J Urol 2002; 167: 516-520.

24. GROSSKLAUS DJ, COFFEY CS et al.: Percent of cancer in the biopsy set predicts pathological findings after prostatectomy. J Urol 2002; 167: 2032-2036.

25. BOCCON-GIBOD L et al.: Percentage of cancer on biopsy cores accurately predicts extracapsular extension and biochemical relapse after radical prostatectomy for T1-T2 prostate cancer. Eur Urol 2000; 37 (4): 449-455.

Dr. F. Osorio Pazo

Hospital de Clínicas "Dr. Manuel Quintelas"

Montevideo (Uruguay)

(Trabajo recibido el 28 marzo de 2003) 